\title{
THE FIGURATRIX IN THE CALCULUS OF VARIATIONS*
}

\author{
BY
}

PAUL R. RIDER

Introduction. In the study of a definite integral of the form

$$
\int_{t_{2}}^{t_{2}} F\left[x(t), y(t), x^{\prime}(t), y^{\prime}(t)\right] d t
$$

Hadamard defines the figurative of the point $(x, y)$ as the curve $F\left(x^{\prime}, y^{\prime}\right)=1$, where $x^{\prime}$ and $y^{\prime}$ are the current coördinates, and $x$ and $y$ are considered constant. $\dagger$ The polar reciprocal of the figurative with respect to the unit circle $x^{\prime 2}+y^{\prime 2}=1$ is termed by Hadamard the figuratrix. $\neq$ The importance of the figuratrix and its space analogue seems to have been overlooked, and it is the desire of the writer to call attention to a number of their interesting properties which enable one to interpret geometrically some of the well known functions and theorems of the calculus of variations.

1. Figurative, figuratrix, and indicatrix. We shall prefer to consider an integral of the form

$$
\int_{t_{1}}^{t_{2}} f[x(t), y(t), \tau(t)]\left(x^{\prime 2}(t)+y^{\prime 2}(t)\right)^{1 / 2} d t
$$

where $\tau=\arctan \left(y^{\prime} / x^{\prime}\right)$. On account of the homogeneity conditions which must be satisfied by the function $F$ in (1), $\S$ we have

$$
F\left(x, y, x^{\prime}, y^{\prime}\right) \equiv F(x, y, \cos \tau, \sin \tau)\left(x^{\prime 2}+y^{\prime 2}\right)^{1 / 2}
$$

and the integral (1) can always be reduced to the form (2). In (2), however, the function $f(x, y, \tau)$ need not be periodic in $\tau$; the right member of equation (3) must be. Moreover, the homogeneity conditions mentioned above do not enter into the consideration of the function $f(x, y, \tau)$. $\|$

- Presented to the Society, December 26, 1924; received by the editors in December, 1924.

† Hadamard, Lȩ̧ons sur le Calcul des Variations, vol. 1, p. 91.

$\ddagger$ Hadamard, loc. cit., p. 92.

$\S F$ must be positive homogeneous of degree one in $x^{\prime}$ and $y^{\prime}$. See Bolza, Vorlesungen über Variationsrechnung, p. 194.

II See Bliss, $A$ new form of the simplest problem of the calculus of variations, these Transactions, vol. 8 (1907), p. 406. This paper will be referred to as Bliss (I). 
Let $P$, of coördinates $x$ and $y$, be a fixed point on an extremal ${ }^{*}$ for the integral (2). Take $P$ as an origin, $p$ and $q$ as rectangular coördinates, and consider the envelope of the variable line $p \cos \tau+q \sin \tau=f(\tau)$. It is readily found that the parametric equations of this envelope are

$$
p=f \cos \tau-f_{\tau} \sin \tau, \quad q=f \sin \tau+f_{\tau} \cos \tau,
$$

functions which play an important rôle in the study of an integral of the form (2). (Subscripts denote partial differentiation.) It is thus easy to identify this envelope with the figuratrix as defined by Hadamard. $\dagger$ For the rectangular coördinates of a point on the figuratrix are $F_{x^{\prime}}\left(x^{\prime}, y^{\prime}\right)$, $F_{y^{\prime}}\left(x^{\prime}, y^{\prime}\right)$. But as a consequence of the homogeneity conditions satisfied by $F$ we have

$$
x^{\prime} F_{z^{\prime}}\left(x^{\prime}, y^{\prime}\right)+y^{\prime} F_{y^{\prime}}\left(x^{\prime}, y^{\prime}\right)=F\left(x^{\prime}, y^{\prime}\right)=F(\cos \tau, \sin \tau)\left(x^{\prime 2}+y^{\prime 2}\right)^{1 / 2},
$$

or

(6) $\quad F_{x^{\prime}}(\cos \tau, \sin \tau) \cos \tau+F_{y^{\prime}}(\cos \tau, \sin \tau) \sin \tau=F(\cos \tau, \sin \tau)=f(\tau)$.

Differentiating, we get

$$
f_{\tau}(\tau)=-F_{x^{\prime}}(\cos \tau, \sin \tau) \sin \tau+F_{y^{\prime}}(\cos \tau, \sin \tau) \cos \tau
$$

Solution of (6) and (7) for $F_{x^{\prime}}$ and $F_{y^{\prime}}$ gives

$$
F_{x^{\prime}}=f \cos \tau-f_{\tau} \sin \tau, F_{y^{\prime}}=f \sin \tau+f_{\tau} \cos \tau .
$$

The arguments of the functions $F_{x^{\prime}}$ and $F_{y^{\prime}}$ may be either $\left(x^{\prime}, y^{\prime}\right)$ or $(\cos \tau$, $\sin \tau$ ), since these functions are homogeneous of degree zero with respect to $x^{\prime}$ and $y^{\prime}$. A comparison of (4) and (8) shows that the envelope of the line $p \cos \tau+q \sin \tau=f(\tau)$ is Hadamard's figuratrix.

If we define the polar figurative as the locus

$$
\rho=f(\tau),
$$

it is seen that the polar figurative is the pedal of the figuratrix with respect to the origin $P$.

The indicatrix $\S$ is the curve

$$
\rho=\frac{1}{f(\tau)} .
$$

* See Bolza, loc. cit., pp. 32, 203.

$\dagger$ It may be remarked that the rectangular equation of the figuratrix is of the form $B(x, y$, $p, q)=0$, where $B$ is a function satisfying the canonical form of Euler's equations in the calculus of variations. See Hadamard, loc. cit., pp. 151-153.

$\ddagger$ See Bolza, loc. cit., pp. 194-197.

$\S$ See Bolza, loc. cit., p. 247. 
Since $f(\tau)=F(\cos \tau, \sin \tau)=F\left(x^{\prime}, y^{\prime}\right) /\left(x^{\prime 2}+y^{\prime 2}\right)^{1 / 2}$, equation (10) is equivalent to the equation $F\left(x^{\prime}, y^{\prime}\right)=1$. That is, the indicatrix is the same as the (rectangular) figurative.

It also follows that

The polar figurative and the indicatrix are inverse curves* with respect to the circle $p^{2}+q^{2}=1$;

The figuratrix and the indicatrix are reciprocal polars $\dagger$ with respect to the circle $p^{2}+q^{2}=1$.

In Fig. 1, $P$ is the origin (a point on an extremal), $Q$ is a point on the figuratrix, $N$ is a point on the polar figurative, $N Q$ is tangent to the figuratrix.

Since the figuratrix and the indicatrix are reciprocal polars, the polar of $Q$ (call it $M R$ ) is tangent to the indicatrix at $M$. This tangent line $M R$, however, gives the direction which is transverse to the direction $P N . \ddagger$

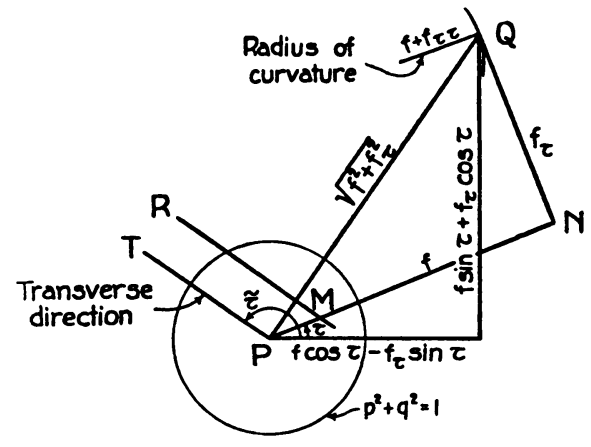

FIG. 1

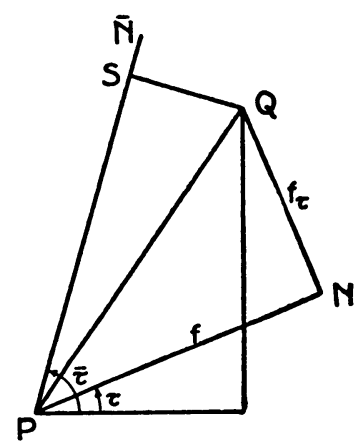

FIG. 2

Thus, the direction $\tilde{\tau}$ which is transverse to $\tau$, or $P N$, is the direction $P T$ which is perpendicular to $P Q$.

This also follows from the formula $\S$ which gives the direction $\tilde{\tau}$ transverse to $\tau$, viz. :

$$
f \cos (\tilde{\tau}-\tau)+f_{\tau} \sin (\tilde{\tau}-\tau)=0 .
$$

For a reference to Fig. 1 or Fig. 2 shows that the left member of (11) is simply the projection of $P Q$ upon the line whose direction is $\tilde{\tau}$. When

* See Williamson, Differential Calculus, 1899, p. 225.

† See Williamson, loc. cit., p. 228.

$\ddagger$ See Bolza, loc. cit., p. 304.

$\S$ See Bliss (I), p. 413. 
this projection is zero the line having the direction $\tilde{\tau}$ is perpendicular to $P Q$.

The normal to the polar figurative is the line joining the point $N$ with the middle point of the line $P Q .^{*}$

2. Geometric interpretations. It can be shown without difficulty that $P Q=\left(f^{2}+f_{r}^{2}\right)^{1 / 2}$. Consequently $N Q=f_{r}$, since $P N=f$. The radius of curvature of the figuratrix at $Q$ is easily computed to be $f+f_{r r}$, whitch is the important $f_{1}$-function for an integral of type (2).

The Weierstrass $e$-function for the integral (2) is

$$
e(x, y, \tau, \bar{\tau})=f(\bar{\tau})-\left[f(\tau) \cos (\bar{\tau}-\tau)+f_{\tau}(\tau) \sin (\bar{\tau}-\tau)\right] .
$$

If, in Fig. 2, $Q$ is a point on the figuratrix, it is seen immediately that the term in square brackets in (12) is $P S$, the projection of $P Q$ upon the line making an angle $\bar{\tau}$ with the polar axis. If $P \bar{N}=f(\bar{\tau})$, the $e$-function is the line-segment $S \bar{N}$. If the integral (2) is to be minimized, we must have $e(x, y, \tau, \bar{\tau}) \geqq 0$ for the set of values $(x, y, \tau)$ giving the point $P$ and the direction of the extremal at that point, and for all values of $\bar{\tau}$. $\dagger$ Consesequently, the projection of $P Q$ upon the line $\tau=\bar{\tau}$ cannot be greater than $f(\bar{\tau})$.

If $d$ is the distance from the tangent line to the figuratrix at $Q(\tau), i$. e. $p \cos \tau+q \sin \tau-f(\tau)=0$, to the point $\bar{Q}(\bar{\tau})$ on the curve, then

$$
\begin{aligned}
d & \left.=\left[f(\bar{\tau}) \cos \bar{\tau}-f_{\tau}(\bar{\tau}) \sin \bar{\tau}\right] \cos \tau+\left[f^{-}\right) \sin \bar{\tau}+f_{\tau}(\bar{\tau}) \cos \bar{\tau}\right] \sin \tau-f(\tau) \\
& =f(\bar{\tau}) \cos (\bar{\tau}-\tau)-f_{\tau}(\bar{\tau}) \sin (\bar{\tau}-\tau)-f(\tau) .
\end{aligned}
$$

Expanding in powers of $(\bar{\tau}-\tau)$ we find that

$$
\begin{aligned}
d & =-\frac{1}{2}(\bar{\tau}-\tau)^{2}\left[f(\tau)+f_{\tau \tau}(\tau)\right]+h \\
& =-\frac{1}{2}(\bar{\tau}-\tau)^{2} f_{1}+h .
\end{aligned}
$$

A similar expansion gives

Thus

$$
\begin{aligned}
e(\tau, \bar{\tau}) & =f(\bar{\tau})-\left[f(\tau) \cos (\bar{\tau}-\tau)+f_{\tau}(\tau) \sin (\bar{\tau}-\tau)\right] \\
& =\frac{1}{2}(\bar{\tau}-\tau)^{2}\left[f(\tau)+f_{\tau \tau}(\tau)\right]+j \\
& =\frac{1}{2}(\bar{\tau}-\tau)^{2} f_{1}+j .
\end{aligned}
$$

$$
e(\tau, \bar{\tau})=-d+k
$$

- See Goursat-Hedrick, Mathematical Analysis, vol. 1, p. 69.

$\uparrow$ See Bliss (I), p. 412. 
The quantities $h, j, k$ are homogeneous of degree three in $(\bar{\tau}-\tau)$ and contain partial derivatives of $f$ with respect to $\tau$ to an order not exceeding the fourth. If these derivatives are finite, $d$ and $e$ are infinitesimals of the second order with respect to $(\bar{\tau}-\tau)$. The geometric significance of $e$ is apparent from (14). The function $e(\tau, \bar{\tau})$ is the distance from the point $\bar{Q}(\bar{\tau})$ on the figuratrix to the tangent line at the point $Q(\tau)$, except for an infinitesimal of at least the third order with respect to $(\bar{\tau}-\tau)$. Under the assumption $f_{1} \geqq 0,{ }^{*}$ this distance is positive $\dagger$ for points $\bar{Q}$ near $Q$, and consequently the figuratrix is concave toward the origin $P$ at the point $Q(\tau)$.

A well known theorem due to Hedrick states that all integrand functions for which the transversality condition is equivalent to orthogonality are of the form $F\left(x, y, x^{\prime}, y^{\prime}\right)=G(x, y)\left(x^{\prime 2}+y^{\prime 2}\right)^{1 / 2}$. In $\S 1$ we found that the direction transverse to $P N(\tau)$ in Fig. 1 is $P T(\tilde{\tau})$, which is perpendicular to $P Q$. But if transversality is to be orthogonality, that is, if $P T$ is to be perpendicular to $P N$, then $P N$ and $P Q$ must coincide. This of course means that the figuratrix, which it will be remembered is the envelope of the variable line $N Q$, must coincide with the polar figurative (locus of $N$ ) and that both curves must be circles. Therefore the equation of the polar figurative must be independent of $\tau$, which means that the function $f(x, y, \tau)$ must be of the form $g(x, y)$. Thus we have a simple proof of Hedrick's theorem.

We shall now investigate the case of discontinuous solutions. $\ddagger$ Let $P_{1} P P_{2}$ be an extremal having a corner at the point $P$. Let $\tau$ be the direction of $P_{1} P$ at $P$, and $\bar{\tau}$ the direction of $P P_{2}$ at $P$. Then at $P$ the following relations, called corner conditions, must be satisfied:§

$$
\begin{aligned}
& f(\tau) \cos \tau-f_{\tau}(\tau) \sin \tau=f(\bar{\tau}) \cos \bar{\tau}-f_{\tau}(\bar{\tau}) \sin \bar{\tau}, \\
& f(\tau) \sin \tau+f_{\tau}(\tau) \cos \tau=f(\bar{\tau}) \sin \bar{\tau}+f_{\tau}(\bar{\tau}) \cos \bar{\tau} .
\end{aligned}
$$

If we refer to (4), the parametric equations for the figuratrix, we see that the above corner conditions simply state that for the corner $P$ the directions $\tau$ and $\bar{\tau}$ give the same point $Q$ on the figuratrix. This is to be expected, since corresponding to a corner point on the extremal we have a double tangent to the indicatrix, $\|$ and the figuratrix is the reciprocal polar of the indicatrix.

* The function $f_{1}$ must be $\geqq 0$ if the integral in (2) is to be minimized. See Bliss (I), p. 409 . † Since it is the negative of $d$.

¥ See Bolza, loc. cit., chapter 8.

\$ See Rider, A note on discontinuous solutions in the calculus of variations, B ull $\mathrm{e} t$ in of th $\mathrm{e}$ American Mathematical Society, vol. 23 (1917), p. 239.

II See Bolza, loc. cit., p. 369. 
It follows that the directions which are transverse to $\tau$ and $\bar{\tau}$ at the corner $P$ coincide, ${ }^{*}$ since the transverse direction is obtained by drawing a perpendicular to $P Q$.

Bliss has defined generalized angle $\nmid$ and generalized area $†$ as

$$
\int \frac{f(\tilde{\tau})}{f^{2}(\tau)}\left(f^{2}(\tau)+f_{\tau}^{2}(\tau)\right)^{1 / 2} d \tau, \quad \iint f(\widetilde{\tau})\left(f^{2}(\tau)+f_{\tau}^{2}(\tau)\right)^{1 / 2} d x d y,
$$

respectively, in which $\tilde{\tau}$ is the direction transverse to $\tau$. It may be worth mentioning that if in Fig. 1 we mark off on $P T$ a length $P \tilde{N}$ equal to $f(\tilde{\tau})$, then the integrand of the second integral in (15) is the area of the rectangle whose sides are $P Q$ and $P \tilde{N}$, and the integrand of the first integral is the ratio of the area of this rectangle to the area of the square whose side is $P N$.

3. The figuratrix in space. For an integral of the form

$$
\int_{t_{1}}^{t_{2}} F\left(x, y, z, x^{\prime}, y^{\prime}, z^{\prime}\right) d t
$$

in which $x, y, z$ are functions of $t$, the figurative of the point $(x, y, z)$ is defined $\S$ as the surface $F\left(x^{\prime}, y^{\prime}, z^{\prime}\right)=1$. The figuratrix is defined as the polar reciprocal of this surface with respect to the unit sphere $x^{\prime 2}+y^{\prime 2}+z^{\prime 2}=1$. We shall, however, deal with the integral\|

$$
\int_{t_{1}}^{t_{2}} f(x, y, z, \tau, \sigma)\left(x^{\prime 2}+y^{\prime 2}+z^{\prime 2}\right)^{1 / 2} d t
$$

where $\tau$ and $\sigma$ are defined by the equations

$$
\begin{aligned}
& x^{\prime} /\left(x^{\prime 2}+y^{\prime 2}+z^{\prime 2}\right)^{1 / 2}=\cos \tau \cos \sigma, \\
& y^{\prime} /\left(x^{\prime 2}+y^{\prime 2}+z^{\prime 2}\right)^{1 / 2}=\sin \tau \cos \sigma, \\
& z^{\prime} /\left(x^{\prime 2}+y^{\prime 2}+z^{\prime 2}\right)^{1 / 2}=\sin \sigma .
\end{aligned}
$$

The geometric significance of $\tau$ and $\sigma$ is at once evident. If at any point of a space curve the positive tangent is constructed, then $\tau$ is the angle which this tangent line makes with its own projection in the $x y$-plane, and $\sigma$ is the angle which this projection makes with the $x$-axis. The figuratrix will be defined as the envelope of the two-parameter family of planes

$$
p \cos \tau \cos \sigma+q \sin \tau \cos \sigma+r \sin \sigma=f(\tau, \sigma),
$$

* Cf. Bolza, loc. cit., p. 369.

$\dagger$ Bliss, $A$ generalization of the notion of angle, these Transactions, vol. 7 (1906), pp. 184-196.

$\ddagger$ Bliss, Generalizations of geodesic curvature and a theorem of Gauss concerning geodesic triangles, American Journal of Mathematics, vol. 37 (1915), pp. 1-18.

$\S$ See Hadamard, loc. cit., p. 96.

II Cf. Rider, The space problem of the calculus of variations in terms of angle, American Journal of Mathematics, vol. 39 (1917), pp. 241-256. This paper will be referred to as Rider (II). 
$p, q, r$ being rectangular coördinates with respect to an origin $P$ on an extremal.

As before, this envelope can be proved identical with Hadamard's figuratrix. In this section we shall develop some of its properties, which are perhaps even more interesting than those of the figuratrix in two dimensions. Where the generalization from two to three dimensions is quite obvious, proofs will be omitted.

By differentiating equation (19) partially with respect to $\tau$ and $\sigma$ we obtain the equations

$$
\begin{aligned}
& -p \sin \tau \cos \sigma+q \cos \tau \cos \sigma=f_{\tau}(\tau, \sigma), \\
& -p \cos \tau \sin \sigma-q \sin \tau \sin \sigma+r \cos \sigma=f_{\sigma}(\tau, \sigma) .
\end{aligned}
$$

Solving equations (19) and (20) for $p, q, r$ (this can be done if $\cos \sigma \neq 0$ ), we get*

$$
\begin{aligned}
& p=f \cos \tau \cos \sigma-f_{\tau} \sin \tau / \cos \sigma-f_{\sigma} \cos \tau \sin \sigma, \\
& q=f \sin \tau \cos \sigma+f_{\tau} \cos \tau / \cos \sigma-f_{\sigma} \sin \tau \sin \sigma, \\
& r=f \sin \sigma+f_{\sigma} \cos \sigma,
\end{aligned}
$$

the rectangular coördinates of a point $Q$ on the figuratrix. It is readily verified that $P Q=\left(p^{2}+q^{2}+r^{2}\right)^{1 / 2}=\left(f^{2}+f_{\tau}^{2} / \cos ^{2} \sigma+f_{\sigma}^{2}\right)^{1 / 2}$.

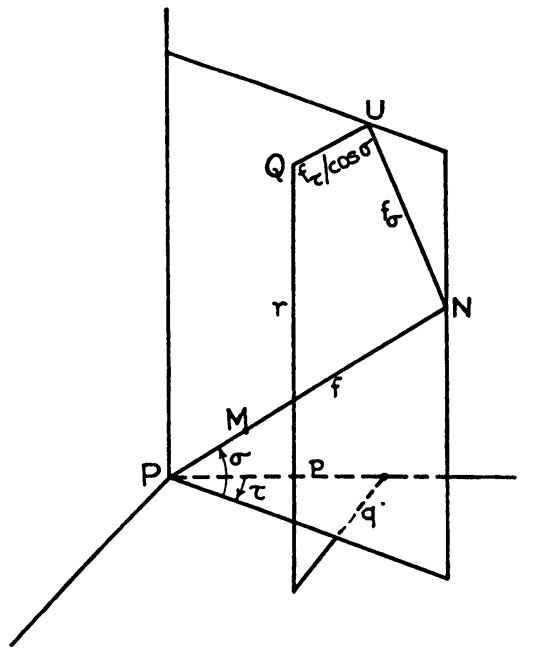

FIG. 3

Let $P N=f(\tau, \sigma), P M=1 / f(\tau, \sigma)$. (See Fig. 3.) Draw $N U=f_{\sigma}$ making an angle of $\pi / 2$ with $P N$ and lying in the plane of $P N$ and the $r$-axis. Then

- Cf. Rider (II), p. 243, equations (5). 
the line-segment $U Q$ will be parallel to the $p q$-plane, perpendicular to $N U$, and equal in length to $f_{\tau} / \cos \sigma$. Moreover

The polar figurative is the pedal surface of the figuratrix with respect to the origin $P$;

The indicatrix is identical with the (rectangular) figurative;

The polar figurative and the indicatrix are inverse surfaces with respect to the sphere $p^{2}+q^{2}+r^{2}=1$;

The figuratrix and the indicatrix are reciprocal polars with respect to the sphere $p^{2}+q^{2}+r^{2}=1$;

The plane $N U Q$ is of course tangent at $Q$ to the figuratrix; the plane through $M$ perpendicular to $P Q$ is tangent to the indicatrix at $M$;

The normal to the polar figurative is the line joining the point $N$ with the middle point of the line $P Q$.

The direction $P T(\tilde{\tau}, \tilde{\sigma})$ which is transverse to $P N(\tau, \sigma)$ is given by the equation*

$$
\begin{gathered}
f(\cos \tau \cos \sigma \cos \tilde{\tau} \cos \tilde{\sigma}+\sin \tau \cos \sigma \sin \tilde{\tau} \cos \tilde{\sigma}+\sin \sigma \sin \tilde{\sigma}) \\
+f_{\tau}\left(\frac{\cos \tau}{\cos \sigma} \sin \tilde{\tau} \cos \tilde{\sigma}-\frac{\sin \tau}{\cos \sigma} \cos \tilde{\tau} \cos \tilde{\sigma}\right) \\
-f_{\sigma}(\cos \tau \sin \sigma \cos \tilde{\tau} \cos \tilde{\sigma}+\sin \tau \sin \sigma \sin \tilde{\tau} \cos \tilde{\sigma}-\cos \sigma \sin \tilde{\sigma})=0 .
\end{gathered}
$$

Since the direction cosines of $P T(\tilde{\tau}, \tilde{\sigma})$ are $\cos \tilde{\tau} \cos \tilde{\sigma}, \sin \tilde{\tau} \cos \tilde{\sigma}, \sin \tilde{\sigma}$, and those of $P Q$ are proportional to $p, q, r$ of (21), it is seen that the direction $P T(\tilde{\tau}, \tilde{\sigma})$ which is transverse to the direction $P N(\tau, \sigma)$ is perpendicular to $P Q$.

It follows at once that if transversality means orthogonality, the function $f(x, y, z, \tau, \sigma)$ must be of the form $g(x, y, z)$.

The parameters of the surface (21) are $\tau$ and $\sigma$ and the fundamental quantities of the first order are

$$
E=p_{\tau}^{2}+q_{\tau}^{2}+r_{\tau}^{2}, \quad F=p_{\tau} p_{\sigma}+q_{\tau} q_{\sigma}+r_{\tau} r_{\sigma}, \quad G=p_{\sigma}^{2}+q_{\sigma}^{2}+r_{\sigma}^{2} .
$$

The functions $p_{r}, q_{r}, r_{\tau}, p_{\sigma}, q_{\sigma}, r_{\sigma}$ are the quantities $-a_{1},-a_{2},-a_{3}-b_{1}$, $-b_{2},-b_{3}$, respectively, found in an earlier paper by the writer, $\dagger$ and it is not difficult to show that

* Cf. Rider (II), p. 250.

$\dagger$ Rider (II), pp. 243-244. 
where

$$
\begin{aligned}
& E=\left(f_{\tau} \tan \sigma+f_{\tau \sigma}\right)^{2} / \cos ^{2} \sigma+\left(f+f_{\sigma \sigma}\right)^{2} \\
& F=\left(f_{\tau} \tan \sigma+f_{\tau \sigma}\right)\left[\left(f+f_{\sigma \sigma}\right)+\left(f-f_{\sigma} \tan \sigma+f_{\tau \tau} / \cos ^{2} \sigma\right)\right] \\
& G=\left(f_{r} \tan \sigma+f_{\tau \sigma}\right)^{2}+\cos ^{2} \sigma\left(f-f_{\sigma} \tan \sigma+f_{\tau \tau} / \cos ^{2} \sigma\right)^{2} \\
& H=\left(E G-F^{2}\right)^{1 / 2}= \pm f_{1} / \cos \sigma
\end{aligned}
$$

\section{If}

$$
f_{1}=\left(f \cos ^{2} \sigma-f_{\sigma} \sin \sigma \cos \sigma+f_{\tau \tau}\right)\left(f+f_{\sigma \sigma}\right)-\left(f_{\tau} \tan \sigma+f_{\tau \sigma}\right)^{2} .
$$

$$
X=\frac{1}{H}\left|\begin{array}{ll}
q_{\tau} & r_{\tau} \\
q_{\sigma} & r_{\sigma}
\end{array}\right|, \quad Y=\frac{1}{H}\left|\begin{array}{ll}
r_{\tau} & p_{\tau} \\
r_{\sigma} & p_{\sigma}
\end{array}\right|, \quad Z=\frac{1}{H}\left|\begin{array}{ll}
p_{\tau} & q_{\tau} \\
p_{\sigma} & q_{\sigma}
\end{array}\right|,
$$

then $X=\cos \tau \cos \sigma, Y=\sin \tau \cos \sigma, Z=\sin \sigma$. This result is to be expected; for $X, Y, Z$ are the direction cosines of the normal to the figuratrix, and the plane (19) is tangent to the figuratrix. The fundamental coefficients of the second order for the figuratrix are

$$
\begin{aligned}
D & =X p_{\tau \tau}+Y q_{\tau \tau}+Z r_{\tau \tau}=-\left(X_{\tau} p_{\tau}+Y_{\tau} q_{\tau}+Z_{\tau} r_{\tau}\right) \\
& =-\left(f \cos ^{2} \sigma-f_{\sigma} \sin \sigma \cos \sigma+f_{\tau \tau}\right), \\
D^{\prime} & =X p_{\tau \sigma}+Y q_{\tau \sigma}+Z r_{\tau \sigma}=-\left(X_{\tau} p_{\sigma}+Y_{\tau} q_{\sigma}+Z_{\tau} r_{\sigma}\right) \\
& =-\left(X_{\sigma} p_{\tau}+Y_{\sigma} q_{\tau}+Z_{\sigma} r_{\tau}\right)=-\left(f_{\tau} \tan \sigma+f_{\tau \sigma}\right), \\
D^{\prime \prime} & =X p_{\sigma \sigma}+Y q_{\sigma \sigma}+Z r_{\sigma \sigma}=-\left(X_{\sigma} p_{\sigma}+Y_{\sigma} q_{\sigma}+Z_{\sigma} r_{\sigma}\right) \\
& =-\left(f+f_{\sigma \sigma}\right) .
\end{aligned}
$$

The total curvature of the figuratrix is $\left(D D^{\prime \prime}-D^{\prime 2}\right) / H^{2}=\cos ^{2} \sigma / f_{1}$. Since for a regular problem $f_{1}$ must be positive, ${ }^{*}$ the figuratrix is a surface of positive curvature at $Q$.

It can be shown that if the integral (17) is to be minimized or maximized the function $f_{1}$ cannot be negative. If we assume $f_{1}>0$, then $D$ and $D^{\prime \prime}$ must be negative for a minimum and positive for a maximum. $\dagger$

The Weierstrass $e$-function for the integral (17) is $\ddagger$

$$
\begin{aligned}
& e(\tau, \sigma, \bar{\tau}, \bar{\sigma},)=f(\bar{\tau}, \bar{\sigma}) \\
& -[f(\tau, \sigma,)(\cos \tau \cos \sigma \cos \bar{\tau} \cos \bar{\sigma}+\sin \tau \cos \sigma \sin \bar{\tau} \cos \bar{\sigma}+\sin \sigma \sin \bar{\sigma}) \\
& \quad+\frac{f_{\tau}(\tau, \sigma)}{\cos \sigma} \cos \bar{\sigma}(-\sin \tau \cos \bar{\tau}+\cos \tau \sin \bar{\tau}) \\
& \quad+f_{\sigma}(\tau, \sigma)(-\cos \tau \sin \sigma \cos \bar{\tau} \cos \bar{\sigma}-\sin \tau \sin \sigma \sin \bar{\tau} \cos \bar{\sigma} \\
& \quad+\cos \sigma \sin \bar{\sigma})] .
\end{aligned}
$$

- See Rider (II), p. 249.

† See Rider (II), p. 249.

† See Rider (II), p. 247. 
But $P S$, the projection of the line $P Q$ upon the line whose direction is $(\bar{\tau}, \bar{\sigma})$, is equal to

$$
p \cos \bar{\tau} \cos \bar{\sigma}+q \sin \bar{\tau} \cos \bar{\sigma}+r \sin \bar{\sigma},
$$

in which $p, q, r$ are given by (21). It is not difficult to show that the expression (23) is the same as that in the square brackets in (22). Therefore, if $P \bar{N}=f(\bar{\tau}, \bar{\sigma})$ is marked off on the line whose direction is given by $(\bar{\tau}, \bar{\sigma})$, the $e$-function is the line segment $S \bar{N}$. If the integral (17) is to be made a minimum, we must have $e(x, y, z, \tau, \sigma, \bar{\tau}, \bar{\sigma}) \geqq 0$ for the set of values $(x, y, z, \tau, \sigma)$ giving the point $P$ and the direction of the extremal at that point, and for all values of $\bar{\tau}$ and $\bar{\sigma} .^{*}$ Consequently, the projection of $P Q$ upon the line $\tau=\bar{\tau}, \sigma=\bar{\sigma}$ cannot be greater than $f(\bar{\tau}, \bar{\sigma})$.

The $e$-function may be written in the form $\dagger$

$$
e(\tau, \sigma, \bar{\tau}, \bar{\sigma})=-\frac{1}{2}\left[(\bar{\tau}-\tau)^{2} D+2(\bar{\tau}-\tau)(\bar{\sigma}-\sigma) D^{\prime}+(\bar{\sigma}-\sigma)^{2} D^{\prime \prime}\right]+h .
$$

Thus, $\ddagger$ the e-function is the distance from the point $\bar{Q}(\bar{\tau}, \bar{\sigma})$ on the figuratrix to the tangent plane at the point $Q(\tau, \sigma)$, except for an infinitesimal of at least the third order with respect to $(\bar{\tau}-\tau)$ and $(\bar{\sigma}-\sigma)$.

Since the $e$-function must be positive for a minimum of the integral (17), the figuratrix is concave at $Q$ toward the origin $P$.

The statements regarding discontinuous solutions admit of obvious generalization from two to three dimensions.

4. Generalization to $n$ dimensions. For the sake of completeness we shall generalize for the case of $n$ dimensions. Let us consider the integral

$$
\int_{t_{1}}^{t_{2}} f\left(x_{1}, \cdots, x_{n}, \tau_{1}, \cdots, \tau_{n-1}\right)\left(x_{1}^{\prime 2}+\cdots+x_{n}^{\prime 2}\right)^{1 / 2} d t,
$$

in which the $x$ 's and $\tau$ 's are functions of $t$. The quantity $\tau_{n-1}$ is the angle made by the positive tangent to a curve with its own projection in the $(n-1)$-space $\left(x_{1}, \cdots, x_{n-1}\right), \tau_{n-2}$ is the angle made by this projection with its own projection in the $(n-2)$-space $\left(x_{1}, \cdots, x_{n-2}\right)$, and so on. Analytically the $\tau$ 's are defined by the equations

$$
\tau_{i}=\arctan \left(x_{i+1}^{\prime} /\left(x_{1}^{\prime 2}+\cdots+x_{i}^{\prime 2}\right)^{1 / 2}\right)
$$$$
(i=1, \cdots, n-1) \text {, }
$$

- See Rider (II), p. 248.

$\dagger$ See Rider (II), p. 248.

† See Eisenhart, Differential Geometry, p. 114.

$\$$ Cf. Rider, On the problem of the calculus of variations in $n$ dimensions, To hok u Mathematical Journal, vol. 13 (1918), pp. 165-171. This paper will be referred to as Rider (III). 
from which it is seen that $x_{i}^{\prime} /\left(x_{1}^{\prime 2}+\cdots+x_{n}^{\prime 2}\right)^{1 / 2}=\sin \tau_{i-1} \cos \tau_{i} \cdots \cos \tau_{n-1}(i=1, \cdots, n-1)$

(if we make the convention $\sin \tau_{0}=1$ ).

Let us now take as origin a point $P\left(x_{1}, \cdots, x_{n}\right)$ on an extremal for the integral (24), and as coördinates the variables $p_{1}, \cdots, p_{n}$. We shall define the figuratrix of the point $P$ as the envelope of the variable hyperplane

$$
\sum_{i=1}^{n} p_{i} \sin \tau_{i-1} \cos \tau_{i} \cdots \cos \tau_{n-1}=f\left(\tau_{1}, \cdots, \tau_{n-1}\right) .
$$

Differentiating partially with respect to $\tau_{1}, \cdots, \tau_{n-1}$, we obtain the following equations:

$$
\begin{aligned}
& -p_{1} \sin \tau_{1} \cos \tau_{2} \cdots \cos \tau_{n-1}+p_{2} \cos \tau_{1} \cdots \cos \tau_{n-1}=f_{\tau_{1}}, \\
& -p_{1} \cos \tau_{1} \sin \tau_{2} \cos \tau_{3} \cdots \cos \tau_{n-1}-p_{2} \sin \tau_{1} \sin \tau_{2} \cos \tau_{3} \cdots \cos \tau_{n-1} \\
& +p_{3} \cos \tau_{3} \cdots \cos \tau_{n-1}=f_{\tau_{3}} \text {, } \\
& -p_{1} \cos \tau_{1} \cdots \cos \tau_{n-2} \sin \tau_{n-1}-\cdots-p_{n-1} \sin \tau_{n-2} \sin \tau_{n-1} \\
& +p_{n} \cos \tau_{n-1}=f_{\tau_{n-1}} \text {. }
\end{aligned}
$$

If $\cos \tau_{2} \cos ^{2} \tau_{3} \cdots \cos ^{n-2} \tau_{n-1}$, the determinant of the coefficients of the system of equations composed of (27) and (28), is different from zero we can solve for the p's obtaining

$$
\begin{aligned}
p_{i}= & f \sin \tau_{i-1} \cos \tau_{i} \cdots \cos \tau_{n-1}+f_{\tau_{i-1}} \frac{\cos \tau_{i-1}}{\cos \tau_{i} \cdots \cos \tau_{n-1}} \\
& -\sum_{j=i}^{n-1} f_{\tau_{i}} \frac{\sin \tau_{j} \sin \tau_{i-1} \cos \tau_{i} \cdots \cos \tau_{j-1}}{\cos \tau_{i+1} \cdots \cos \tau_{n-1}} \quad(i=1, \cdots, n),
\end{aligned}
$$

the rectangular coördinates of a point $Q$ on the figuratrix.

As in the preceding cases, the direction which is transverse to the direction $\left(\tau_{1}, \cdots, \tau_{n-1}\right)$ is orthogonal to $P Q$. $^{*}$

Since the normal to the figuratrix is parallel to the line $P N$, the direction cosines of this normal are

(30) $X_{i}=x_{i}^{\prime} /\left(x_{1}^{\prime 2}+\cdots+x_{n}^{\prime 2}\right)^{1 / 2}=\sin \tau_{i-1} \cos \tau_{i} \cdots \cos \tau_{n-1}(i=1, \cdots, n)$.

\footnotetext{
- For the transversality condition see Rider (III), pp. 168-169.
} 
The fundamental coefficients of the second order for the figuratrix are then defined as

$$
D_{j k}=-\sum_{i=1}^{n} \frac{\partial X_{i}}{\partial \tau_{j}} \frac{\partial p_{i}}{\partial \tau_{k}}
$$

The partial derivatives occurring in $D_{j k}$ are found to be

$$
\begin{aligned}
& \frac{\partial X_{i}}{\partial \tau_{j}}=-\sin \tau_{i-1} \cos \tau_{i} \cdots \cos \tau_{j-1} \sin \tau_{i} \cos \tau_{j+1} \cdots \cos \tau_{n-1} \quad(j \geqq i), \\
& \frac{\partial X_{i}}{\partial \tau_{i-1}}=\cos \tau_{i-1} \cos \tau_{i} \cdots \cos \tau_{n-1} \text {, } \\
& \frac{\partial X_{i}}{\partial \tau_{j}}=0 \\
& \frac{d p_{i}}{d \tau_{j}}=-\frac{\sin \tau_{j} \sin \tau_{i-1} \cos \tau_{i} \cdots \cos \tau_{j-1}}{\cos \tau_{j+1} \cdots \cos \tau_{n-1}}\left(f \cos ^{2} \tau_{j+1} \cdots \cos ^{2} \tau_{n-1}\right. \\
& \left.-\sum_{k=j+1}^{n-1} f_{\tau_{k}} \sin \tau_{k} \cos \tau_{k} \cos ^{2} \tau_{j+1} \cdots \cos ^{2} \tau_{k-1}+f_{\tau_{j} \tau_{j}}\right) \\
& +\frac{\cos \tau_{i-1}}{\cos \tau_{i} \cdots \cos \tau_{n-1}}\left(f_{r_{i-1}} \tan \tau_{j}+f_{\tau_{i-1} r_{j}}\right) \\
& -\sum_{k=i}^{i-1} \frac{\sin \tau_{k} \sin \tau_{i-1} \cos \tau_{i} \cdots \cos \tau_{k-1}}{\cos \tau_{k+1} \cdots \cos \tau_{n-1}}\left(f_{\tau_{k}} \tan \tau_{j}+f_{\tau_{\tau^{r}} r_{j}}\right) \\
& -\sum_{k=j+1}^{n-1} \frac{\sin \tau_{k} \sin \tau_{i-1} \cos \tau_{i} \cdots \cos \tau_{k-1}}{\cos \tau_{k+1} \cdots \cos \tau_{n-1}}\left(f_{\tau_{j}} \tan \tau_{k}+f_{\tau_{j} \tau_{k}}\right)(j \geqq i), \\
& \frac{d p}{d \tau_{i-1}}=\frac{\cos \tau_{i-1}}{\cos \tau_{i} \cdots \cos \tau_{n-1}}\left(f \cos ^{2} \tau_{i} \cdots \cos ^{2} \tau_{n-1}\right. \\
& \left.-\sum_{k=i}^{n-1} f_{\tau_{k}} \sin \tau_{k} \cos \tau_{k} \cos ^{2} \tau_{i} \cdots \cos ^{2} \tau_{k-1}+f_{r_{i-1} \tau_{i-1}}\right) \\
& -\sum_{k=i}^{n-1} \frac{\sin \tau_{k} \sin \tau_{i-1} \cos \tau_{i} \cdots \cos \tau_{k-1}}{\cos \tau_{k+1} \cdots \cos \tau_{n-1}}\left(f_{\tau_{i-1}} \tan \tau_{k}+f_{\tau_{i-1} \tau_{k}}\right) \text {, } \\
& \begin{aligned}
\frac{d p_{i}}{d \tau_{j}}= & \frac{\cos \tau_{i-1}}{\cos \tau_{i} \cdots \cos \tau_{n-1}}\left(f_{\tau_{j}} \tan \tau_{i-1}+f_{\tau_{j} \tau_{i-1}}\right) \\
& -\sum_{k=i}^{n-1} \frac{\sin \tau_{k} \sin \tau_{i-1} \cos \tau_{i} \cdots \cos \tau_{k-1}}{\cos \tau_{k+1} \cdots \cos \tau_{n-1}}\left(f_{\tau_{j}} \tan \tau_{k}+f_{\tau_{j} \tau_{k}}\right)(j<i-1) .
\end{aligned}
\end{aligned}
$$


It is found that

$$
\begin{array}{ll}
D_{i i}=-( & \cos ^{2} \tau_{i+1} \cdots \cos ^{2} \tau_{n-1}-\sum_{j=i+1}^{n-1} f_{\tau_{j}} \sin \tau_{j} \cos \tau_{j} \cos ^{2} \tau_{i+1} \cdots \cos ^{2} \tau_{j-1} \\
& \left.+f_{\tau_{j \tau_{j}}}\right), \\
D_{i j}=-\left(f_{\tau_{i}} \tan \tau_{j}+f_{\tau_{i} \tau_{j}}\right) & (i \neq j) .
\end{array}
$$

(It is assumed that $f \cos ^{2} \tau_{i+1} \cdots \cos ^{2} \tau_{n-1}=f$ when $i=n-1$.) The writer has already shown that for a minimum of the integral (24) the quantities $D_{i i}$ cannot be positive.*

Let us define the quantity $B$ by means of the formula

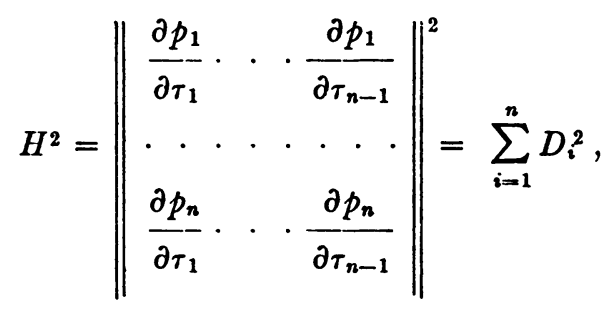

where $D_{i}$ is the determinant obtained by omitting the $i$ th row from the matrix. Then $\dagger$

$$
D_{i}=(-1)^{i-1} f_{1} X_{i} / \cos \tau_{2} \cos ^{2} \tau_{3} \cdots \cos ^{n-2} \tau_{n-1},
$$

where $f_{1}$ is the symmetric determinant

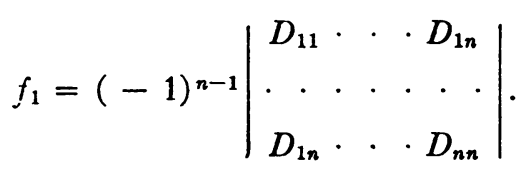

Thus

$$
H^{2}=f_{1}^{2} /\left(\cos \tau_{2} \cos ^{2} \tau_{3} \cdots \cos ^{n-2} \tau_{n-1}\right)^{2} .
$$

The total curvature of the figuratrix is

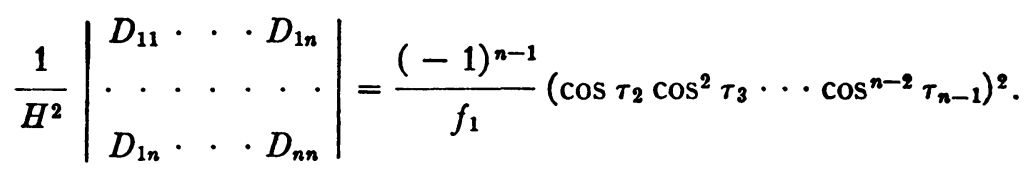

- See Rider (III), p. 171. In that paper $\alpha_{i j}$ is the negative of $D_{i j}$.

$\dagger$ See Rider, On the $f_{1}$-function in the calculus of variations, Washington University Studies, vol. 5, scientific series no. 2 (Jan., 1918), p. 100. 
The Weierstrass $e$-function is the function*

$$
\begin{aligned}
& e\left(\tau_{1}, \cdots, \tau_{n-1}, \bar{\tau}_{1}, \cdots, \bar{\tau}_{n-1}\right)=f\left(\bar{\tau}_{1}, \cdots, \bar{\tau}_{n-1}\right) \\
&- \sum_{i=1}^{n} p_{i} \sin \bar{\tau}_{i-1} \cos \bar{\tau}_{i} \cdots \cos \bar{\tau}_{n-1} \\
&=-\frac{1}{2}\left[\sum_{i=1}^{n-1}\left(\bar{\tau}_{i}-\tau_{i}\right)^{2} D_{i i}+\sum_{i=1, j=i+1}^{n-1}\left(\bar{\tau}_{i}-\tau_{i}\right)\left(\bar{\tau}_{i}-\tau_{j}\right) D_{i j}\right]+h,
\end{aligned}
$$

the $p$ 's being defined by (29). It thus appears, that with proper restrictions on the partial derivatives of $f$ which occur in $h$, the e-function is the distance from the point $\bar{Q}$ on the figuratrix to the tangent hyperplane at $Q$ except for an infinitesimal of at least the third order with respect to the quantities $\left(\bar{\tau}_{i}-\tau_{i}\right)$. The concavity of the figuratrix toward the origin $P$ follows, since $e$ must be positive for a minimum value of the integral (24).

- See Rider (III), pp. 169-170.

WASHINGTON UNIVERSITY

ST. Lours, Mo. 